way or another, it is then the question how to deal with these uncertainties. This is why we propose to make the assessment as transparent as possible and distinguish environmental aspects from other aspects, strive to apply the best authorized scientific knowledge available and advice to perform sensitivity analyses in order to see to what extent uncertainties influence the final results. With respect to the loss of information, we think that is precisely the other way around. Information can be gained by performing an impact assessment. The inventory table data have not disappeared but they are interpreted which can give new (and not less) point of views. An impact assessment can also be performed per process and not only for the entire product system which enables a new range of analysis techniques, particularly with respect to the identification of improvement options (cf. 6).

Last but not least, lca as we described it in this paper only deals with the environmental aspects of a product without mixing these with other aspects such as financial, technical and macro-political aspects (e.g. third world issues). This does not mean that we think these aspects are not important. On the contrary, these are very important too, but we think for a transparent overall valuation it is much more appropriate to distinguish these different issues clearly. A pilot study distinguishing between the assessment of environmental and micro-economic aspects of investments in a joint framework has just been published (15). Leiden (Netherlands)

\section{References}

1) Consoli, F., D. Allen, I. Boustead, N. de Oude, J. Fava, W. Franklin, B. Quay, R. Parrish, R. Perriman, D. Postlethwaite, J. Seguin and B. Vigon, 1993: Guidelines for Life-Cycle Assessment: A >Code of Practice (Edition 1). setacEurope, Brussels.

2) Heijungs, R., J.B. Guinée, G. Huppes, R.M. Lankreijer, H. A. Udo de Haes, A. Wegener Sleeswijk, A. A.M. Ansems, P. G. Eggels, R. van Duin and H.P. de Goede, 1992: Environmental life cycle assessment of products. Guide \& Backgrounds October 1992. cml, Leiden.

3) Guinée, J.B., H.A. Udo de Haes and G. Huppes, 1993: Quantitative Life Cycle Assessment of Products: Goal Definition and Inventory. J. Cleaner Prod. Vol. 1, No. 1, pp. 3-13.

4) Guinée, Jeroen B., Heijungs, Reinout, Udo de Haes, Helias A. and Huppes, Gjalt (1993): Quantitative life cycle assessment of products: 2. Classification, valuation and improvement analysis. J. Cleaner Prod. Vol. 1 No. 2, pp. 8191.

5) Guinée, J.B. and Heijungs, R., 1993: Classification factors for toxic substances within the framework of life cycle assessment of products. Chemosphere, Volume 26, Number 10, 19251944.

6) Heijungs, R., 1993: A generic method for the identification of options for cleaner products. Accepted for publication in Ecological Economics.
7) Guinée, J. B., 1993: Data for the Normalization Step within Life Cycle Assessment of Products. cml-paper no. 14, cml, Leiden.

8) bus, 1984: Ökobilanzen von Packstoffen. Schriftenreihe Umweltschutz no. 24. Bundesamt für Umweltschutz.

9) Mekel, O.C. L., G. Huppes, R. Huele, \& J. B. Guinée, 1990: Environmental effects of different package systems for fresh milk. $\mathrm{cml}$ report 70, Leiden.

10) Life-Cycle Assessment. Proceedings of SETAC-Europe workshop on Environmental Life Cycle Assessment of Products, December 2-3 1991 in Leiden. setac-Europe, Brussels

11) Wenzel, H. and M. Hauschild, 1993: Key issues for toxicity assessment of a product life cycle. Paper presented at the setac/secotox workshop on ecotoxicity Lyngby January 7-8 1993, Technical University of Denmark, Lyngby, 14 pp.

12) Anonymous, 1994: Eco-indicator project. Developing the eco-indicator as a decision support tool for product development. Duijf Consultancy bv, Vught.

13) Lindeijer, E. W., M. Sprengers, A. L. W. van Roekel, J.G.M. Kortman, H.J.W. Sas, A.L. Viergever \& G. J. Teesink, 1993: In preparation. ides \& ce, Amsterdam/Delft. 14) Heijungs, R., 1994: Maatschappelijke weging van milieuproblemen. $\mathrm{cml}$, Leiden.

15) Wit, R., H. Taselaar, R. Heijungs and G. Huppes, 1993: reim: lca-based ranking of environmental investment options. cml-report 103, $\mathrm{cml}$, Leiden.

\title{
Standardisierung von Produkt-Ökobilanzen
}

\section{Zur Begründung von Konventionen und Standardisierungen}

Die Ergebnisse der bisher vorgelegten Ökobilanzen weichen - national und international stark voneinander ab, je nach der gewählten Methodik, dem zugrundegelegten Bilanzraum und den angewandten Daten. Beispielhaft soll auf Ökobilanzen für Fensterrahmen, Windelanwendungen und Verpackungen verwiesen werden.

Um Ergebnisse von Ökobilanzen für gesamtgesellschaftlich bedeutsame umweltorientierte $\mathrm{Be}$ wertungen und Entscheidungen zu nutzen, müssen sie auf konsensfähigen und tragfähigen $\mathrm{Me}$ thoden basieren. Diese Arbeiten können nur als »Gemeinschaftsarbeit« aller beteiligten Kreise organisiert werden, zumal die Festlegung des Bilanzraumes und insbesondere die Bewertung nicht auf objektiven, wissenschaftlich ermittelten Wahrheiten sondern auf Konventionen beruhen werden. Sie werden daher zugleich von dem »Wollen « der Beteiligten getragen, sich un- abhängig oder unter Zurückstellung von Einzelinteressen zu einigen. Dies ist zugleich die Begründung, diese Arbeiten aufgrund des dort geltenden Konsensprinzips im Rahmen der Normung zu organisieren.

Aufgrund der immer stärkeren europäischen und auch internationalen Ausrichtung des produktbezogenen Umweltschutzes (Europäischer Binnenmarkt, globale Umweltthemen) sollten zudem international gültige Konventionen unter Berücksichtigung nationaler Besonderheiten angestrebt werden, zumal die wissenschaftliche Methodendiskussion bereits sehr stark internationalisiert ist (SETAC-Aktivitäten).

\section{Stand der Institutionalisierung der Arbeiten}

1993 konnten sowohl international als auch national wichtige Weichen für den Aufbau von Strukturen zur Erarbeitung entsprechender Konventionen gestellt werden. Im Rahmen der ISO (Internationale Normungsorganisation) wurde das Sub-Committee 5 »Life Cycle As- sessment« im ISO-TC 207 Environmental Management (Sekretariat: Frankreich, Vorsitz: Deutschland, Dr. Marsmann, Bayer AG) eingerichtet. Die Aufgabenstellung lautet: »Standardization in the field of life cycle assessment as a tool for environmental management of product and service systems. It encompasses the assessment of impacts on the environment from the extraction of raw material to the final disposal of waste « (Resolution 6/1993 ISO/TC 207/SC 5). Wie bei entsprechenden Arbeiten üblich, hat man sich hier in Anlehnung an das Standardmodell produktbezogener Ökobilanzen in Arbeitsgruppen aufgeteilt:

- WG 1 »Life cycle assessment - general working principles and procedures" (USA); Scope: The ISO WG 1 (..) is committed to develop principles and guidelines to harmonize the practice for undertaking and reporting LCA studies in a responsible transparent and consistent manner. (...) (cooperation with other WG's) (Resolution 7/1993).

- WG 2 »Life cycle inventory analysis - general (Deutschland) 
- WG 3 »Life cycle inventory analysis - specific « (Japan); Scope: Development of methodology to perform and report Life Cycle Inventory (Resolution 8/1993)

-WG 4 "Life cycle impact assessment (Schweden); Scope: Development of generally agreed methodological principles for the assessment of the environmental impacts of product and service systems based on life cycle inventory data (Resolution 9/1993).

- WG 5 "Life cycle improvement assessment « (F); Scope: The WG $5(\ldots)$ is committed to developing guidelines and harmonizing practices to help identify and evaluate opportunities that minimize the environmental burdens (related to data from life cycle inventory analysis) or impacts (related to data from life cycle impact assessment) of products and services which provide the same function (Resolution 10/1993).

Bei den weiteren internationalen Aktivitäten ragen die bereits 1991 begonnenen Arbeiten der SETAC (Society of Environmental Toxicology and Chemistry) heraus, in deren Rahmen im August 1993 die Veröffentlichung eines »Code of Practice, Guidelines for Life Cycle Assessments « erfolgte (1). Zu erwähnen sind ferner diverse Aktivitäten anderer internationaler Organisationen:

- OECD (Pollution Prevention and Control Group PPCG),

- ECE (Environmental Product Profiles),

- UNEP (Promotion Cleaner Products),

- EG/EU (Environmental Labelling),

- CEN (Life Cycle Assessments for Packaging).

Bei einer derartigen Vielzahl von Aktivitäten sind Überschneidungen oder auch widersprüchliche Entwicklungen nicht zu vermeiden. Um so bedeutsamer ist die Schaffung konsensgetragener Strukturen wie bei der ISO zu bewerten. Hinzu kommen Aktivitäten anderer Staaten, insbesondere der Niederlande, USA, Kanada, Frankreich, Schweiz, den Nordischen Ländern (Schweden, Dänemark) und Großbritannien.

National stehen in diesem Zusammenhang die 1993 auch offiziell aufgenommenen Arbeiten des DIN/NAGUS-Arbeitsausschuß 3 ProduktÖkobilanzen im Vordergrund, der zugleich (als nationaler Spiegelausschuß des Sub-Committees "Life Cycle Analysis«) im Rahmen des ISO TC 207) arbeitet. Folgende Arbeitsgruppen wurden eingerichtet:

- Grundsätze produktbezogener Ökobilanzen,

- Methodik der Sachbilanzen,

- Wirkungsbilanz und Bewertung.

Als erstes Arbeitsergebnis werden im MärzHeft der DIN-Mitteilungen die Grundsätze produktbezogener Ökobilanzen (Stand Oktober 1993) veröffentlicht, mit dem eine nationale Verständigung über wichtige Begriffe, Standardvorgehensweisen, Grundsätze, Möglichkeiten und Grenzen produktbezogener Ökobilanzen gelang (2). Abgebildet ist das hier ent- haltene »Prozeßschema produktbezogener Ökobilanzen « (siehe Abbildung 1).

Als herausragend hinsichtlich ihrer potentiellen politischen Bedeutung sind die Arbeiten der Enquete-Kommission »Schutz des Menschen und der Umwelt « des Deutschen Bundestages zu bewerten, die sich auch mit dem Thema Ökobilanzen befassen (3). Durch diese Arbeiten und Strukturen wurde eine Bündelung diverser Einzelaktivitäten, z. B. des Verbandes der Chemischen Industrie, der Umweltbewegung um das Öko-Institut Freiburg oder des Umweltbundesamtes (4) erreicht.

\section{Wichtige Prämissen für die Standardisierungsarbeit}

Die Erarbeitung methodischer Regeln für produktbezogene Ökobilanzen wird wesentlich erleichtert, wenn Klarheit über Sinn und Zweck, Möglichkeiten und Grenzen von Ökobilanzen besteht. Ihr wesentlicher Zweck ist die konzeptionelle Umsetzung eines medienübergreifenden ganzheitlichen Ansatzes des produktbezogenen Umweltschutzes mit dem Ziel

- die Umweltbeeinträchtigungen im Lebenslauf der Produkte zu erfassen und zu bewerten, um

- Schwachstellen zu identifizieren,

- Optimierungen (z. B. Roh- und Werkstoffe, Produktgestaltung, Transport, Entsorgung) vorzunehmen und

- alternative (konkurrierende) Varianten zur Erreichung eines gleichen Nutzens (z.B. Werkstoffe, Verpackungen, Stoffe, Produktvarianten, Entsorgungsvarianten, Dienstleistungen) ökologisch vergleichend zu bewerten

Akteure öffentlich bedeutsamer Ökobilanzen sind gleichermaßen Staat, Wirtschafts-, Verbraucher- und Umweltverbände, Gewerkschaften, politische Parteien und die Wissenschaft. Öffentliche Anwendungen sind z. B.

- Begründung für umweltpolitische Zielvorstellungen im produktbezogenen Umweltschutz (z. B. Verpackungen und Abfall, wirtschaftspolitische Maßnahmen),

- Festsetzung von Abgaben, z. B. umweltbelastungsadäquate $\mathrm{CO}_{2}$-Abgabe für Energieträger,

- Begründung für die Verbraucher- und Umweltberatung,

- Begründung für verbandspolitische Forderungen und Stellungnahmen,

- Begründung für Produktauswahl und Kriterienfindungen im Rahmen von Umweltzeichen (Blauer Engel, Europäisches Umweltzeichen),

- Begründung für Empfehlungen, z. B. in welchen Produktbereichen die Verwendung von nachwachsenden Rohstoffen ökologisch sinnvoll ist.

Historisch gesehen sind jedoch die unternehmensbezogenen Anwendungen Ausgangspunkt der Ökobilanzdiskussion gewesen. Die Industrie hat nach wie vor ein großes Interesse, Ökobilanzen als Entscheidungsgrundlage für ökologische Produktoptimierungen zu nutzen, sowohl produktseitig (z.B. Auswahl der Werkstoffe, Zulieferer, Transportlogistik und Entsorgung), als auch anlagenseitig (z. B. Maßnahmen zur Energieeinsparung, Luft- und Wasserreinhaltung oder Abfallvermeidung). Hinzu kommt die Nutzung von Ökobilanzen in Produktinformation, Marketing und Werbung.

In den weiteren Standardisierungsarbeiten sollten jedoch bestimmte Möglichkeiten und Grenzen produktbezogener Ökobilanzen beachet werden:

1. Ökobilanzen stellen eine bedeutsame methodische Fortentwicklung der Erfassung und Bewertung umweltbezogener Aspekte der Produktgestaltung dar. Sie ergänzen insbesondere die bisher weitgehend sektoral mit Blick auf Einzelaspekte vorherrschenden Betrachtungsweisen, z. B. hinsichtlich bestimmter Gefahrstoffe, Schadstoff- und Lärmemissionen oder Abfallbelastungen.

2. Der Begriff »Ökobilanz« wird derzeit häufig noch zu schlagwortartig und pauschalisierendhäufig ohne Verbindung mit den eingeschränkten jeweils verfolgten Untersuchungszielen - benutzt. Auch bestehen häufig unrealistisch optimistische Erwartungen über den Nutzen dieses Instruments und seiner Anwendbarkeit für die Fortentwicklung des Umweltschutzes. Ferner besteht die Gefahr von Entscheidungsschwächen oder eines überzogenen zweckgeleiteten Pessimismus, der darauf abzielt, mit dem Hinweis auf die fehlende wissenschaftliche Fundierung einzelner Ökobilanzen notwendige und sinnvolle Umweltschutzmaßnahmen zu verzögern.

3. Ökobilanzen können politische und ökonomische Entscheidungen vorbereiten, stützen und präzisieren, aber nicht ersetzen, zumal ergänzend zu den umweltbezogenen Auswirkungen auch ökonomische, soziale und andere Aspekte in der Entscheidungsfindung zu berücksichtigen sind.

4. Eine wissenschaftlich gestützte Bewertung von Konflikten zwischen konkurrierenden Umweltschutzzielsetzungen (z. B. Luftreinhaltung lokal/atmosphärisch, Abfallverwertung/Bodenund Gewässerschutz) durch Ökobilanzen ist nur ökobilanzspezifisch im Kontext der Verständigung über eine allgemein anerkannte Bewertungsmethode zu erwarten.

5. Eine objéktive und wissenschaftlich gestützte Bewertung unterschiedlicher Ergebnisse ist auch mittel- und langfristig nicht zu erwarten. Andererseits ist eine Konvention über eine allgemein anerkannte Bewertungsmethode für die Fortentwicklung der Ökobilanzen unabdingbar 6. Bei jeder Ökobilanz stellt sich ein Zielkonflikt zwischen dem Entscheidungsbedarf über Abschneidungen einerseits und der Aufstellung methodisch idealtypischer Ökobilanzen andererseits.

Zusammenfassend kann festgehalten werden Ökobilanzen sind kein l'art pour l'art, sondern 
sollen ökologisch geprägte Antworten auf bestimmte Fragestellungen möglich machen. Modellbildungen sollten aufgrund der sehr unterschiedlichen Fragestellungen und Anwendungszwecke von daher nicht »für sich « erfolgen, Detailschärfe anstreben oder starr verfaßt werden, sondern sich vor allem an dem Sinn von Ökobilanzen und seiner bereits jetzt erkennbaren Möglichkeiten und Grenzen orientieren.

\section{Ausblick: Möglichkeiten und Grenzen der Standardisierung}

1. Generalistisch geprägte Arbeiten/Diskussionen: Aufgrund der völlig unterschiedlichen Themen der Ökobilanzen wird die generell-abstrakt orientierte Methodenentwicklung vor allem die Verständigung über Begriffe, allgemeine Mindestanforderungen, Vorgehensweisen, Optionen und Prioritäten zum Inhalt haben.

2. Die gegen Standardisierungen vorgebrachten Argumente weisen im wesentlichen auf folgende Punkte hin:

- Der erreichte Diskussionsstand läßt Festlegungen noch nicht zu.

- Standardisierungen sind zu starr für eine eher flexibel zu handhabende Bilanzierungs- und Bewertungsmethode.

- In den gewählten Strukturen wird sich die Industrie aufrund der Ehrenamtlichkeit und Eigenfinanzierung der Arbeiten gegenüber den anderen Beteiligten durchsetzen.

3. Aus gegenwärtiger Sicht sind diese Hinweise allesamt nicht zutreffend. Die Entwicklung zumindest der Sachbilanzmethodik ist schon recht weit gediehen, die bisherigen Entwürfe sind durchaus flexibel zu handhaben und setzen eher auf »zu begründende Optionen « als auf einseitige Festlegungen und - zumindest in Deutschland- ist eine für einen Normungsausschuß bemerkenswert breite Pluralität erreicht worden. Dies spiegelt sich auch in den ersten positiven Bewertungen des Grundsatzpapieres (2) wieder. 4. Der SETAC »Code of Practice« (1) dokumentiert in diesem Sinne die Schwierigkeiten theoretischer Methodenentwicklung, da häufig auf Festlegungen verzichtet wird und stattdessen »Optionen « unterbreitet werden. Auf der anderen Seite schafft er eine Kommunikationsbasis für sämtliche einschlägige Arbeiten.

5. Die Konsensbildungsprozesse sind sowohl national und erst recht international als »schwierig « einzuschätzen. Das » Voranbringen « ist dabei auch vom »Operationsgeschick « bzw. der »-taktik« der zuständigen Convener, Obleute und Sekreteriate abhängig.

6. Auch bei der angestrebten Internationalität der Methodik werden spezifische Ökobilanzen und Anwendungen aufgrund der national sehr unterschiedlichen Prioritäten der Produkt- und Umweltthemen, des Schutzniveaus und der Daten (?) vor allem national erarbeitet und diskutiert werden.

7. Der Methodenentwicklung mangelt es bisher an der eigentlich unabdingbaren Verzahnung zwischen »Theorie « und »Praxis«. Die derzei- tigen Arbeiten des Umweltbundesamtes zur Methodik der Wirkungsbilanz und der Bewertung sind daher bewußt anwendungsorientiert an den Ergebnissen der Ökobilanz Verpackungen ausgerichtet worden. Der zwischenzeitlich erreichte Diskussionsstand läßt dabei einen vorsichtigen Optimismus zu, tatsächlich zu einheitlichen Vorgehensweisen zu kommen.

8. Die Normungsarbeit sollte auch eher im Umfeld der Ökobilanzen anzusiedelnde Themen einbeziehen, z. B. Anforderungen an die Beteiligung der Fachöffentlichkeit, und die Präsentation. Ein weiteres Thema ist die Verständigung auf einen standardisierten Berichtsbogen (Kurzfassung von Ökobilanzen), der von den Bilanzierern selbst oder extern erstellt wird.

Harald Neitzel, Berlin

\section{Anmerkungen}

(1) SETAC (Society for Environmental Toxicology and Chemistry), A »Code of Pactice«, Guidelines for Life-Cycle Assessment, August 1993; anzufordern bei SETAC Europe, Avenue E. Mounier 83, Box 1, B-1200 Brussels, Tel. 32277272 81; Fax 322 7705386

(2) Dieses Grundsatzpapier wurde im Zeitraum September 1992 bis Oktober 1993 in insgesamt 10 Entwürfen erarbeitet. Die Veröffentlichung ist Anfang 1994 mit dem Titel: »DIN/NAGUS-Arbeitssausschuß 3 Produkt-Okobilanzen, Grundsätze Produktbezogener Ökobilanzen, Stand Oktober 1993 « in den DIN Mitteilungen vorgesehen (siehe Abbildung 1).

(3) Zwischenbericht der Enquete-Kommission "Schutz des Menschen und der Umwelt - Bewertungskriterien und Perspektiven für umweltverträgliche Stoffkreisläufe in der Industriegesellschaft « des 12. Deutschen Bundestages, Bonn 1993

(4) Umweltbundesamt: Ökobilanzen für Produkte, Bedeutung, Sachstand, Perspektiven, UBA-TEXTE 38/92 (kostenlos anzufordern)

\section{Abbildung 1}

\section{Prozeßschema einer produktbezogenen Ökobilanz}

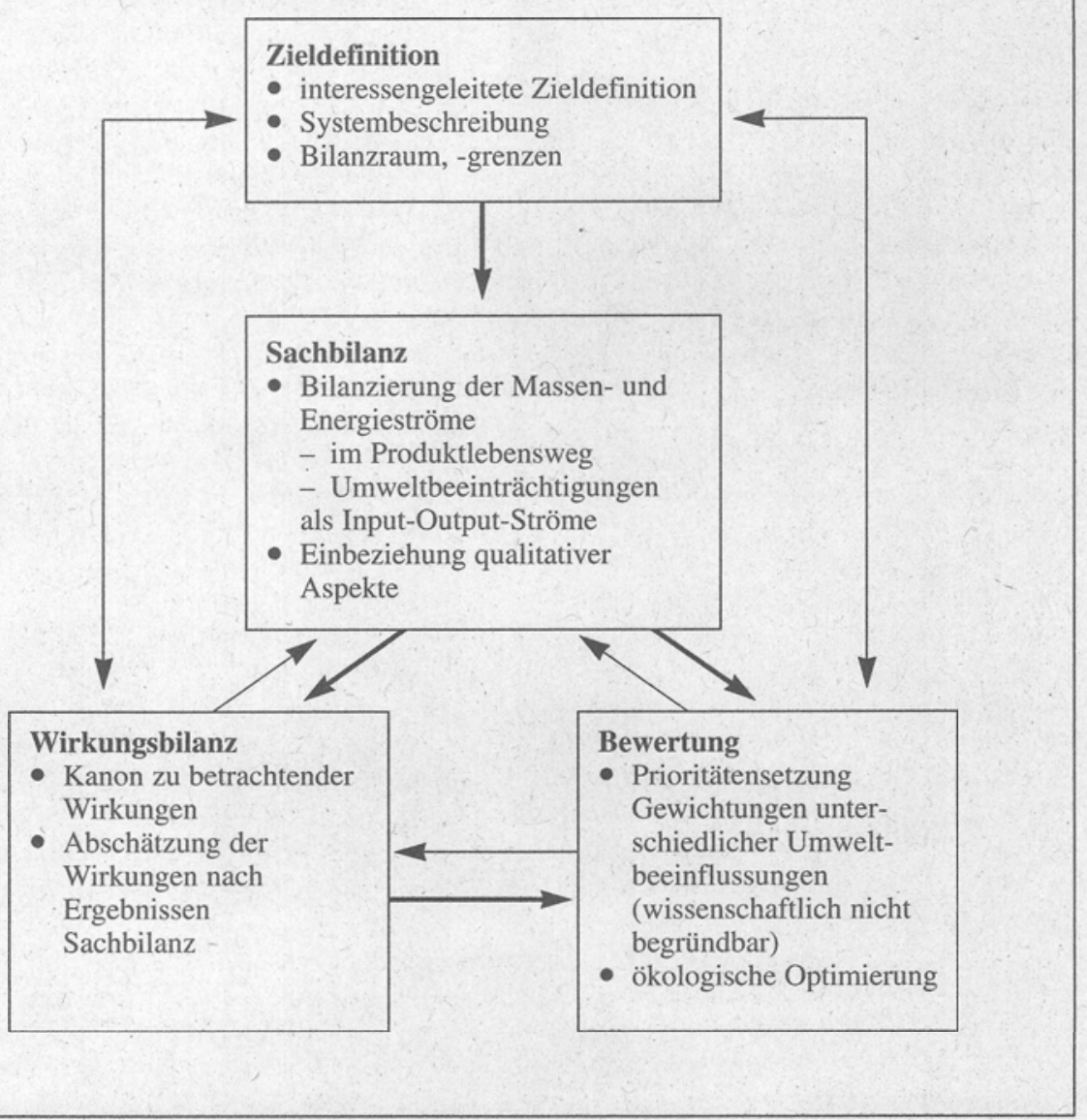


(c) 20I0 Authors; licensee IÖW and oekom verlag. This is an article distributed under the terms of the Creative Commons Attribution Non-Commercial No Derivates License (http://creativecommons.org/licenses/by-nc-nd/3.o/), which permits unrestricted use, distribution, and reproduction in any medium, provided the original work is properly cited. 